Mindera HEALTH

\title{
A DERMAL BIOMARKER PATCH DISPLAYS EXCELLENT ANALYTICAL PERFORMANCE AND OUTPERFORMS TAPE STRIPPING IN PSORIATIC SKIN
}

\author{
Mindera Health, 5795 Kearny Villa Road, San Diego, CA 92123
}

\section{SYNOPSIS}

The Dermal Biomarker Patch (DBP) platform efficiently captures transcriptomes of $>7,000$ biomarkers from the lesional skin of psoriasis patients in sufficient quantities for next-generation sequencing protocols. There was no significant body-site variation observed, in contrast to stratum corneum tape stripping." This platform makes precision medicine in dermatology a reality. It provides a powerful tool for doctors, researchers, and patients to better understand the skin.

\section{OBJECTIVE}

To demonstrate the ability of the Mindera Health DBP to extract actionable quantities of mRNA from lesional skin of psoriasis patients, and explore the body-site dependence of this method.

\section{METHODS}

Using the DBP, a total of 416 transcriptomes were collected from 24 different body areas; each transcriptome was comprised of $~ 7,000$ biomarkers. Samples were collected from research sites $(\mathrm{N}=15)$ under an IRB-approved protocol. After collection, samples were placed in a storage buffer between $2-8^{\circ} \mathrm{C}$ for transport and processing.

Once received, next generation sequencing (NGS) was performed according to standard procedures. Measurements were made of the gene detection rate and mRNA yield. Additionally, a subset of samples was analyzed at various time points after sample collection (1-10 days) to determine mRNA stability during storage and transport.

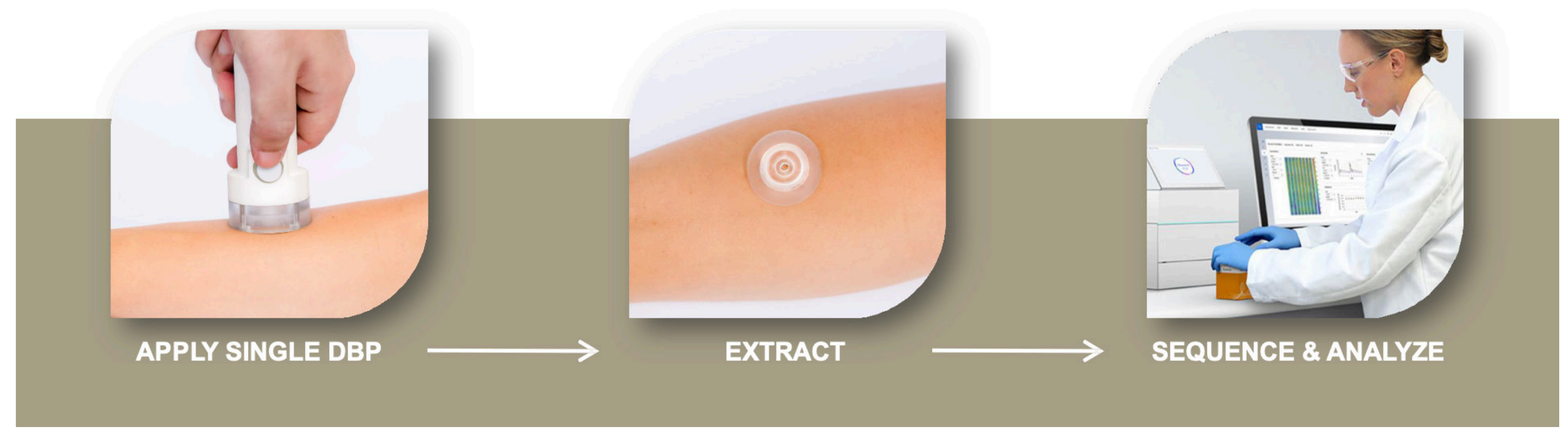

FIGURE 1. Dermal Biomarker Patch workflow.

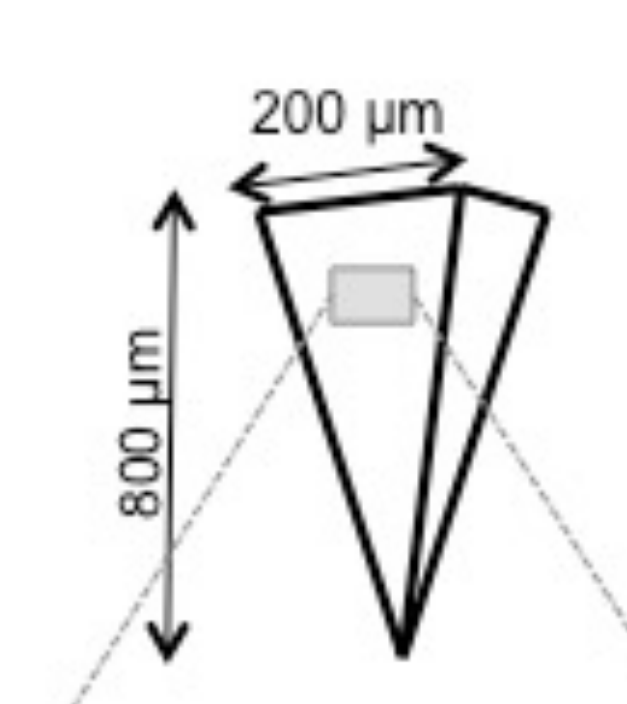

Probe 1 11

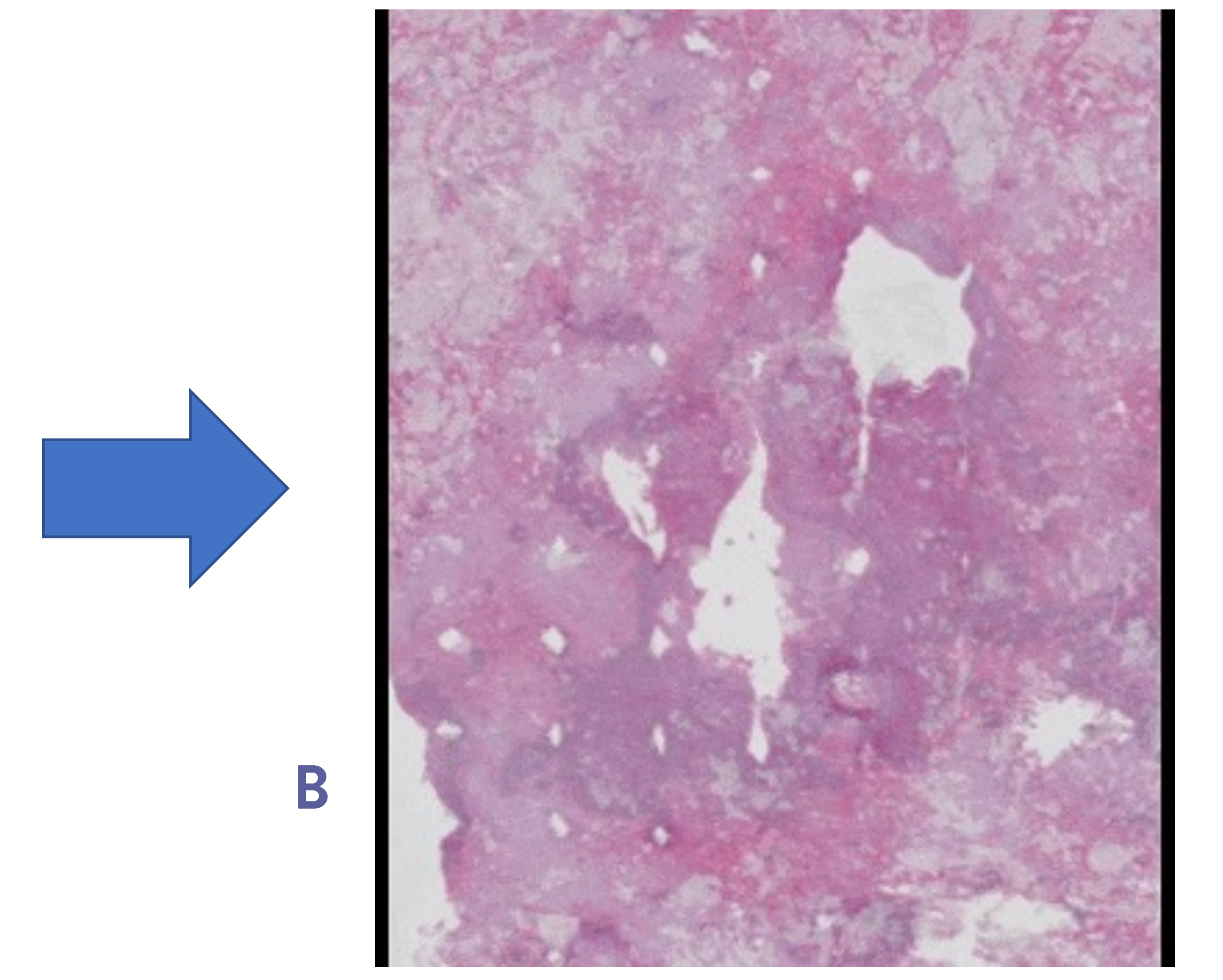

FIGURE 2.(A) Graphical depiction of DBP micro-projections. These projection are chemically modified to specifically (B) En face the depth of penetration by the assess vivo skin samples were sliced en face and On average, $>90 \%$ of the DBP projections On average, $>90 \%$ of the DBP projections resulting puncture sites were funtifed
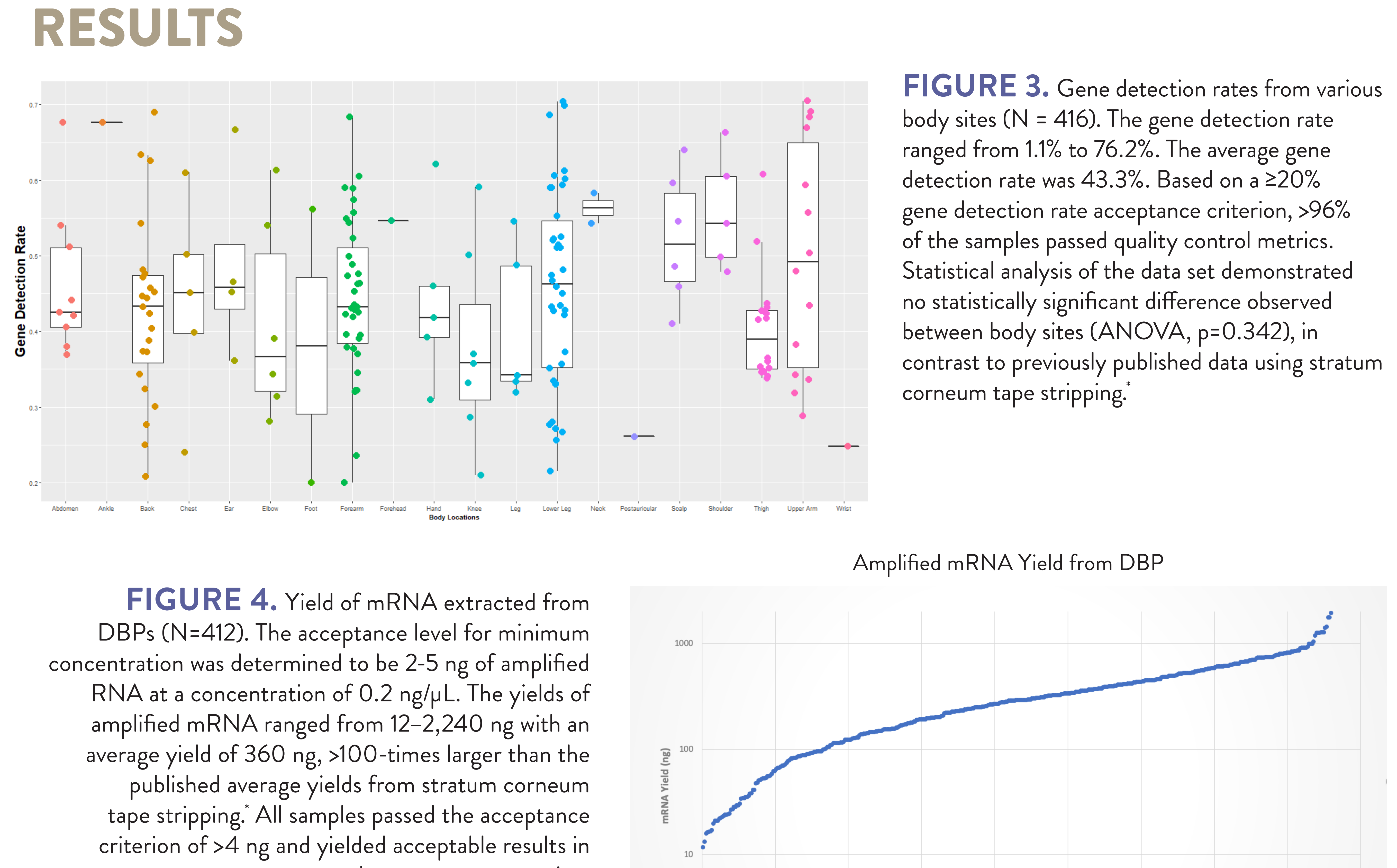
downstream sequr

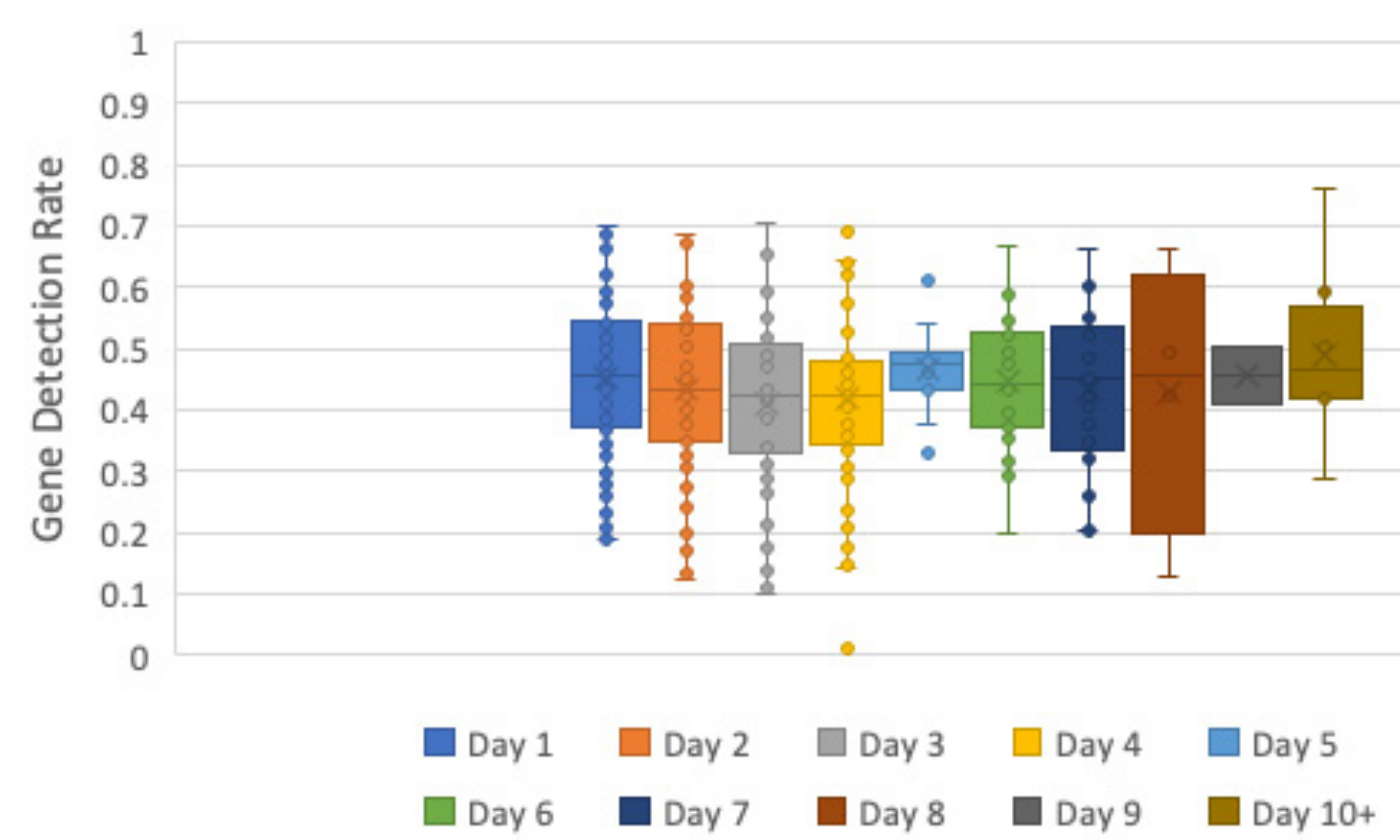
collection and processing on quality of
DBP RNA-Seq data. A subset of 373 psoriasis skin samples was collected from research sites, stored in storage buffer at $2-8^{\circ} \mathrm{C}$, and transported in an insulated shipper system at $2-8^{\circ} \mathrm{C}$ overnight for downstream analysis. To substantiate mRNA stability, the gene detection rates were determined for samples stored from I day to $10+$ days. A total of $94.7 \%$ of the samples exceeded the QC threshold of $\square$ Day 6 Day 7 Day 8 Day $\square$ Day $10+$
FIGURE 5. Influence of time between

\section{CONCLUSION}

The Mindera Health Dermal Biomarker Patch platform has been proven to reliably extract the skin transcriptome in a minimally invasive manner. Success in psoriasis patients includes:

- highly reproducible efficiency of extraction

- excellent mRNA yields suitable for RNA-Seq protocols

- $>96 \%$ of samples passing quality control metrics

- no body-site bias in transcriptome extraction 